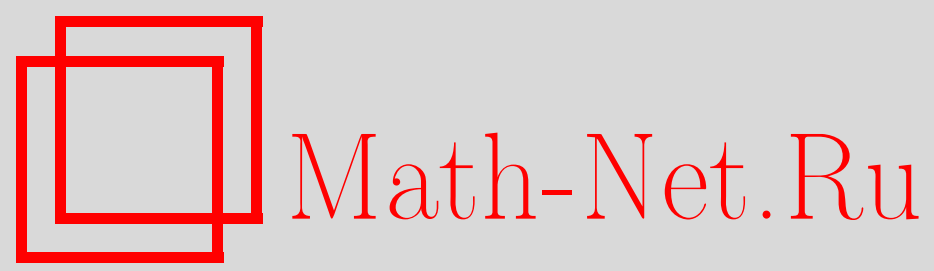

Л. А. Минин, Е. Г. Супонев, Е. А. Киселев, Использование метода моментов для аппроксимации сигналов, содержащих структурные элементы в форме пиков, Вестник ТвГУ. Серия: Прикладная математика, 2020, выпуск 3, 68-77

DOI: https://doi.org/10.26456/vtpmk576

Использование Общероссийского математического портала Math-Net.Ru подразумевает, что вы прочитали и согласны с пользовательским соглашением

http://www.mathnet.ru/rus/agreement

Параметры загрузки:

IP : 3.85 .73 .92

26 апреля 2023 г., $17: 13: 30$ 
УДК 519.651, 004.627

\section{ИСПОЛЬЗОВАНИЕ МЕТОДА МОМЕНТОВ ДЛЯ АППРОКСИМАЦИИ СИГНАЛОВ, СОДЕРЖАЩИХ СТРУКТУРНЫЕ ЭЛЕМЕНТЫ В ФОРМЕ ПИКОВ}

Минин Л.А., Супонев Е.Г., Киселев Е.А.

Воронежский государственный университет, г. Воронеж

Поступила в редакцию 22.07.2019, после переработки 05.10.2020.

В данной работе производится обобщение метода моментов, предложенного в одной из недавних статей для моделирования зубцов электрокардиограммы комплексами из нескольких функций Гаусса. Цель заключалась в том, чтобы сделать метод применимым для функций более общего вида, сохранив простоту его программной реализации. Для этого был проведен ряд математических преобразований в общем виде и получены достаточно простые соотношения для вычисления параметров модельного сигнала. Это дает возможность применять для аппроксимации участков сигналов функции разнообразного вида, продиктованные их физической моделью. Единственным ограничением для используемых функций является существование необходимого количества моментов, а момент нулевого порядка должен быть отличен от нуля. В данной работе продемонстрировано несколько примеров реализации обобщенного метода моментов. Показано, что на практике в зависимости от вида используемой для моделирования функции возникает ряд вычислительных особенностей, касающихся точности метода и его устойчивости по отношению к шуму. Полученные результаты могут быть полезны для разработки новых эффективных моделей биомедицинских сигналов, атомных и ядерных спектров, а также иных типов сигналов, имеющих локальные особенности в форме пиков.

Ключевые слова: метод моментов, цифровой сигнал, выделение компонент, сжатие.

Вестник ТвГУ. Серия: Прикладная математика. 2020. № 3. С. 68-77. https://doi.org/10.26456/vtpmk576

\section{Введение}

В основе многих современных алгоритмов цифровой обработки сигналов лежит разложение по какому-либо базису. В настоящее время весьма популярны вейвлеты различных типов [1] и функции Уолша [2]. Не утратили свою актуальность и разнообразные модификации преобразования Фурье [3].

Теоретически базисы в функциональных пространствах должны состоять из бесконечного числа функций, но на практике используются их конечные наборы. 
При этом с точки зрения задачи сжатия информации, чем меньше функций мы используем для аппроксимации сигнала, тем выгоднее. Например, в ряде публикаций показано, что для моделирования зубцов электрокардиограммы (ЭКГ) достаточно хорошо подходят комплексы из одной [4] или нескольких функций Гаусса [5]. В работе [6], используя это обстоятельство, удалось повысить эффективность сжатия ЭКГ по сравнению с алгоритмами, базирующимися на стандартном применении дискретного преобразования Фурье и всплесков Добеши.

Алгоритм, предложенный в статье [5], основан на использовании начальных моментов $\nu_{n}$

$$
\nu_{n}=\int_{-\infty}^{\infty} x^{n} f(x) d x, n=0,1, \ldots
$$

где $f(x)$ - исследуемый сигнал. Аргумент $x$, если речь идет об ЭКГ, представляет собой время. Интегралы в формуле (1) на практике рассчитываются приближенно по квадратурным формулам с помощью дискретной сетки отсчетов, на которой задан цифровой сигнал.

В статье [5] в качестве модельного сигнала рассматривается комплекс из нескольких функций Гаусса с неопределенными параметрами. Если приравнять несколько первых моментов исследуемого и модельного сигнала, то получается достаточно простая система уравнений для нахождения неизвестных параметров. Хорошая эффективность сжатия сигнала ЭКГ, по сути, достигается за счет удачного выбора модели для зубцов. По этой причине для их аппроксимации требуется всего несколько функций. Для сигналов другого типа, вместо функции Гаусса потребуется использовать иные модели, продиктованные какими-либо физическими соображениями или результатами экспериментов.

Целью данной работы является обобщить метод моментов, предложенный в статье [5], на случай широкого класса функций, сохранив при этом простоту его программной реализации.

\section{1. Теоретическая часть}

Пусть имеется некоторая функция $\varphi(x)$, заданная на всей вещественной оси, у которой существуют моменты (1) до 5-го порядка включительно, причем $\nu_{0} \neq 0$. Из-за наличия множителя $x^{n}$ под интегралом моменты более высокого порядка рассчитываются по квадратурным формулам весьма неточно, поэтому на практике их использовать нецелесообразно.

Рассмотрим сигнал $f(x)$. С помощью функции $\varphi(x)$, взятой с разными амплитудами и локализованной в разных точках, строится модельный сигнал $\widetilde{f}(x)$

$$
\widetilde{f}(x)=\sum_{j=1}^{N} A_{j} \varphi\left(x-m_{j}\right)
$$

где $N$ - общее количество компонент, $A_{j}$ - их амплитуды, $m_{j}$ - сдвиг по аргументу $x$. В статье [5] использовались 3 компоненты в виде функции Гаусса. 
Предположим, что нам известны $2 N$ первых моментов $\nu_{n}$ исследуемого сигнала $f(x)$. Приравняем к ним моменты модельного сигнала (2)

$$
\nu_{n}=\int_{-\infty}^{\infty} x^{n}\left(\sum_{j=1}^{N} A_{j} \varphi\left(x-m_{j}\right)\right) d x, n=0,1, \ldots, 2 N-1
$$

Преобразуем формулу (3): поменяем суммирование и интегрирование местами, а затем в каждом из интегралов сделаем замену переменных $t=x-m_{j}$. В результате имеем:

$$
\nu_{n}=\sum_{j=1}^{N} A_{j} \int_{-\infty}^{\infty}\left(t+m_{j}\right)^{n} \varphi(t) d t=\sum_{j=1}^{N} A_{j} \sum_{k=0}^{n} C_{n}^{k} m_{j}^{k} \int_{-\infty}^{\infty} t^{n-k} \varphi(t) d t
$$

где $C_{n}^{k}$ - биномиальный коэффициент. Если обозначить $I_{k}=\int_{-\infty}^{\infty} t^{k} \varphi(t) d t$, то получим следующую систему уравнений относительно неизвестных $A_{j}$ и $m_{j}$ :

$$
\sum_{j=1}^{N} A_{j} \sum_{k=0}^{n} C_{n}^{k} m_{j}^{k} I_{n-k}=\nu_{n}, n=0,1, \ldots, 2 N-1
$$

Формулу (5) можно существенно упростить. Для этого поменяем порядок суммирования, затем выделим слагаемые, содержащие старшие степени $m_{j}$, а все остальные перенесем в правую часть равенства

$$
I_{0} \sum_{j=1}^{N} A_{j} m_{j}^{n}=\nu_{n}-\sum_{k=0}^{n-1} C_{n}^{k} I_{n-k} \sum_{j=1}^{N} A_{j} m_{j}^{k}, n=0,1, \ldots, 2 N-1 .
$$

Все неизвестные в правой части (6) можно исключить в уравнении с номером $n$, если воспользоваться всеми предыдущими. Предполагая, что $I_{0} \neq 0$, в результате приходим к набору соотношений

$$
\sum_{j=1}^{N} A_{j} m_{j}^{n}=b_{n}, n=0,1, \ldots, 2 N-1,
$$

где величины $b_{n}$ можно считать известными, поскольку они легко вычисляются рекуррентно

$$
b_{0}=\frac{\nu_{0}}{I_{0}}, b_{n}=\frac{1}{I_{0}}\left(\nu_{n}-\sum_{k=0}^{n-1} C_{n}^{k} I_{n-k} b_{k}\right) .
$$

Формула (7) дает $2 N$ уравнений относительно $2 N$ неизвестных $A_{j}$ и $m_{j}$. Кроме того, по структуре она совпадает с системой уравнений, полученной в статье [5] для случая, когда $\varphi(x)$ являлась функцией Гаусса. Разница состоит только в способе вычисления коэффициентов $b_{n}$. Поэтому полностью справедливы все формулы, полученные после этого в работе [5]. Приведем наиболее важные из них. 
При $N=2$ система (7) сводится к квадратному уравнению, корнями которого являются искомые $m_{1}$ и $m_{2}$

$$
\begin{gathered}
m^{2}-q_{1} m-q_{0}=0, \\
q_{1}=\frac{b_{1} b_{2}-b_{0} b_{3}}{b_{1}^{2}-b_{0} b_{2}}, q_{0}=\frac{b_{1} b_{3}-b_{2}^{2}}{b_{1}^{2}-b_{0} b_{2}} .
\end{gathered}
$$

При $N=3$ получается кубическое уравнение

$$
\begin{aligned}
& m^{3}-c_{2} m^{2}-c_{1} m-c_{0}=0, \\
& c_{2}=\alpha\left(b_{3} p_{3}+b_{2} p_{5}-b_{1} p_{6}\right), \\
& c_{1}=\alpha\left(b_{3} p_{2}+b_{2} p_{4}-b_{0} p_{6}\right), \\
& c_{0}=\alpha\left(b_{3} p_{1}+b_{1} p_{4}-b_{0} p_{5}\right),
\end{aligned}
$$

где коэффициенты $\alpha$ и $p_{i}, i=1,2, \ldots, 6$ определяются выражениями

$$
\begin{aligned}
& p_{1}=b_{2}^{2}-b_{1} b_{3}, p_{2}=b_{2} b_{3}-b_{1} b_{4}, \\
& p_{3}=b_{3}^{2}-b_{3} b_{4}, p_{4}=b_{2} b_{4}-b_{1} b_{5}, \\
& p_{5}=b_{2} b_{5}-b_{3} b_{4}, p_{6}=b_{4}^{2}-b_{3} b_{5}, \\
& \alpha=b_{2} p_{1}+b_{1} p_{2}+b_{0} p_{3} .
\end{aligned}
$$

Отыскав $m_{j}$, далее достаточно легко можно найти $A_{j}$, поскольку при заданных $m_{j}$ формула (7) превращается в систему линейных алгебраических уравнений относительно амплитуд $A_{j}$.

\section{2. Вычислительные эксперименты}

Рассмотрим несколько примеров реализации описанного выше алгоритма для функций $\varphi(x)$ различной формы. Ниже представлен наиболее важный в практическом отношении случай $N=3$.

Тестовые сигналы $f(x)$ генерируются следующим образом. Мы выбираем значения трех амплитуд $A_{j}$ и параметров сдвига $m_{j}$. Далее функция $f(x)$ строится в виде суммы компонент $A_{j} \varphi\left(x-m_{j}\right)$ с добавлением случайного шума $\varepsilon(x)$

$$
f(x)=\sum_{j=1}^{3} A_{j} \varphi\left(x-m_{j}\right)+\varepsilon(x) .
$$

После этого мы выбираем дискретный набор точек с некоторым постоянным шагом $h$ и рассчитываем по формуле трапеции моменты $\nu_{n}$ тестового сигнала до 5-го порядка включительно. Затем вычисляем амплитуды и параметры сдвига, используя формулы $(8),(11)-(13)$. Соответствующие значения мы обозначим $\widetilde{A}_{j}$ и $\widetilde{m}_{j}$. Наконец, по формуле $(2)$, используя величины $\widetilde{A}_{j}$ и $\widetilde{m}_{j}$, строим модельный сигнал $\widetilde{f}(x)$.

Для оценки степени соответствия модельного сигнала $\widetilde{f}(x)$ исходному $f(x)$, мы рассчитывали их отклонение $\delta$ друг от друга по среднеквадратичной норме

$$
\delta=\sqrt{\frac{\sum_{m=1}^{M}\left(\tilde{f}\left(x_{m}\right)-f\left(x_{m}\right)\right)^{2}}{M}},
$$


где $M$ - количество дискретных точек, $x_{m}=(m-1) h, m=1,2, \ldots, M$.

На Рис. 1 представлен пример работы алгоритма для функции вида

$$
\varphi(x)=x^{2} \exp \left(-\frac{(x-\Delta)^{2}}{2 \sigma^{2}}\right) .
$$

Функции с подобной структурой применяются для расчета строения и свойств молекулярных систем в квантовой химии [7]. В случае, изображенном на Рис. 1, параметры имели следующие значения: $h=0.25, \Delta=1, \sigma=1, A_{1}=3, A_{2}=10, A_{3}=-5, m_{1}=5, m_{2}=11, m_{3}=14$. Уровень случайного шума составлял в среднем $5 \%$ относительно амплитуды сигнала.

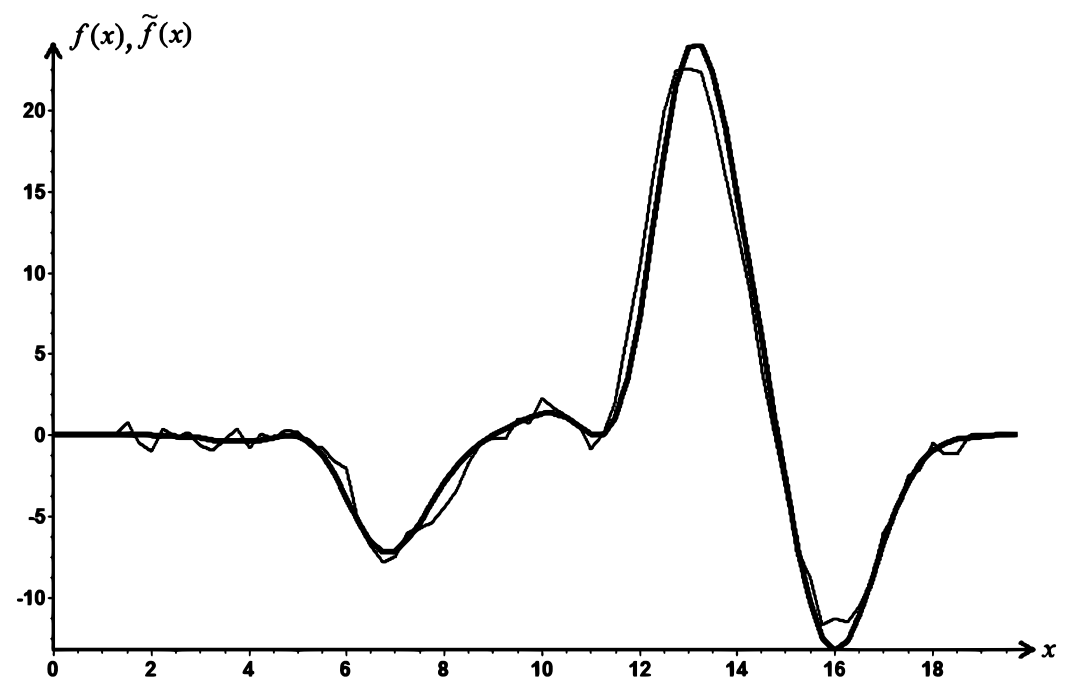

Рис. 1: Исходньй (тонкая линия) и модельный (жирная линия) сигналь

Как показывает Рис. 1, в среднем сигнал восстанавливается достаточно хорошо: отклонение по среднеквадратичной норме значительно меньше амплитуды сигнала и составляет $\delta \sim 1.2$. Однако, из-за наличия шума местоположение второго пика оказалось несколько искажено. Является ли подобное отклонение допустимым, зависит от требуемой точности для каждой конкретной практической задачи. Также отметим, что при достижении шумом уровня свыше $5 \%$ качество восстановления достаточно быстро ухудшается.

На Рис. 2 представлен еще один пример реализации метода моментов, где для расчетов была выбрана другая функция

$$
\varphi(x)=e^{-\sigma|x|} \sin (\omega x+\alpha) .
$$

Сдвиги функций подобного вида с разными частотами представляют собой частный случай так называемых оконных систем [8]. Использовались следующие значения параметров: $h=0.25, \Delta=1, \sigma=2, \omega=0.5, \alpha=\pi / 4, A_{1}=7, A_{2}=-8, A_{3}=-5, m_{1}=3, m_{2}=7$, 


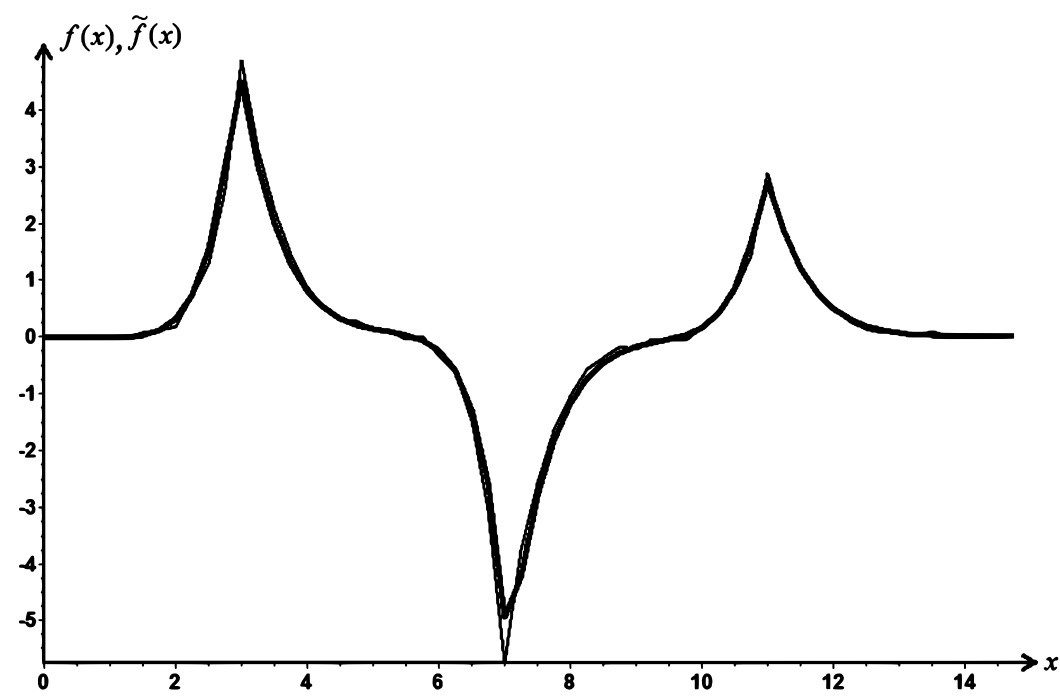

Рис. 2: Исходный (тонкая линия) и модельный (жирная линия) сигналь

$m_{3}=11$. Уровень шума составлял $3 \%$. Сигнал в этом случае в среднем также восстанавливается достаточно надежно: $\delta \sim 0.033$.

Как показывает Рис. 2, местоположения пиков определены с достаточно высокой точностью. Амплитуды найдены с погрешностью $5 \sim 10 \%$. Однако уровень шума, после превышения которого заметно падает качество восстановления сигнала, для функции (17) несколько ниже $(\sim 3 \%)$, чем для (16). Таким образом, в зависимости от выбранной функции $\varphi(x)$, при реализации метода моментов возникает ряд вычислительных особенностей, которые важно учитывать при решении конкретных прикладных задач.

\section{Заключение}

В данной работе было произведено обобщение метода моментов, предложенного в статье [5]. Это дает право использовать для аппроксимации участков сигналов достаточно разнообразные функции $\varphi(x)$, продиктованные какой-либо физической моделью. Для применимости полученных соотношений требуется только существование у функции $\varphi(x)$ необходимого количества моментов, а также, чтобы у нее не был равен нулю момент нулевого порядка.

После обобщения метод по-прежнему остается простым для программной реализации и позволяет при $N \leqslant 4$ получить аналитические формулы для расчета амплитуд и параметров сдвига. Это возможно сделать, применив формулы Кардано и Феррари. Таким образом, цель данной работы можно считать достигнутой.

Если $N>4$ метод моментов приводит к уравнению степени $N$, корни которого можно найти численно, но это лишено практического смысла, поскольку существуют проблемы с вычислением моментов высоких порядков. Кроме того, неортогональные системы сдвигов, состоящие из большого числа функций, проявляют известную неустойчивость, о которой рассказано, например, в статье [9]. 
В перспективе предполагается провести более детальное изучение особенностей реализации метода моментов для различных функций $\varphi(x)$. Также во многих математических моделях в физике используются функции, у которых не существует даже момент первого порядка. Примером является функция Лоренца (распределение Коши или функция Брейта-Вигнера), широко применяемая в атомной спектроскопии [10] и ядерной физике [11]. Для таких случаев можно попытаться модифицировать определение моментов (1), используя какие-либо весовые функции, но это требует отдельного исследования.

\section{Список литературы}

[1] Addison P. Wavelet Transforms and the ECG: a Review // Physiological Measurement. 2005. Vol. 26, № 5. Pp. R155-R199.

[2] Голубов Б.И., Ефимов А.В., Скворцова В.А. Ряды и преобразования Уолша: Теория и применения. М.: Издательство ЛКИ, 2008. 352 с.

[3] Bracewell R. The Fourier Transform and its Applications. New York: McGraw-Hill, 2000. $618 \mathrm{p}$.

[4] Никифоров П.Л. Модель электрокардиографического сигнала на основе совокупности колокольных импульсов // Вестник молодых ученых. Серия: Технические науки. 1998. № 1. С. 64-68.

[5] Насер Н. Аппроксимация пиков в электрокардиограммах комбинацией сдвигов функции Гаусса // Системы управления и информационные технологии. 2015. № 1(59). C. 77-80.

[6] Киселев Е.А., Насер Н.М., Супонев Е.Г. Комбинированный алгоритм сжатия сигнала электрокардиограммы с помощью всплесков Добеши и функции Гаусса // Системы управления и информационные технологии. 2017. № 3(69). C. $53-56$.

[7] Матулис В.Э., Матулис В.Э., Ивашкевич О.А. Прикладная квантовая химия. Минск: Издательство БГУ, 2007. 143 с.

[8] Добеши И. Десять лекций по вейвлетам. Ижевск: НИЦ «Регулярная и хаотическая динамика», 2001. 464 с.

[9] Журавлев М.В., Минин Л.А., Ситник С.М. О вычислительных особенностях интерполяции с помощью целочисленных сдвигов гауссовых функций // Beдомости Белгородского государственного университета. 2009. № 13(68). С. 8999.

[10] Lang K.R. Astrophysical Formulae. Berlin, Heidelberg, New York: Springer Verlag, 1980. $783 \mathrm{p}$.

[11] Мухин К.Н. Экспериментальная ядерная физика. Т. 2. СПб.: Лань, 2009. 326 с. 


\section{Образец цитирования}

Минин Л.А., Супонев Е.Г., Киселев Е.А. Использование метода моментов для аппроксимации сигналов, содержащих структурные элементы в форме пиков // Вестник ТвГУ. Серия: Прикладная математика. 2020. №3. С.68-77. https://doi.org/10.26456/vtpmk576

\section{Сведения об авторах}

\section{1. Минин Леонид Аркадьевич}

доцент кафедры математической физики физического факультета Воронежского государственного университета.

Россия, 394018, г. Воронеж, Университетская пл., 1, ВГУ.

E-mail: mininla@mail.ru

\section{2. Супонев Евгений Геннадьевич}

магистрант факультета компьютерных наук Воронежского государственного университета.

Россия, 394018, г. Воронеж, Университетская пл., 1, ВГУ.

E-mail: suponev7ry@yandex.ru

\section{3. Киселев Евгений Александрович}

ассистент кафедры цифровых технологий факультета компьютерных наук Воронежского государственного университета.

Россия, 394018, г. Воронеж, Университетская пл., 1, ВГУ.

E-mail: evg-kisel2006@yandex.ru 


\title{
USING THE METHOD OF MOMENTS FOR THE APPROXIMATION OF SIGNALS CONTAINING PEAK SHAPED STRUCTURAL ELEMENTS
}

\author{
Minin Leonid Arkad'evich \\ Associate Professor at Mathematical Physics department, \\ Voronezh State University \\ Russia, 394018, Voronezh, Universitetskaya sq., 1, VSU. \\ E-mail: mininla@mail.ru \\ Suponev Evgenii Gennad'evich \\ Graduate student at Computer Science faculty, \\ Voronezh State University \\ Russia, 394018, Voronezh, Universitetskaya sq., 1, VSU. \\ E-mail: suponev777@yandex.ru \\ Kiselev Evgenii Aleksandrovich \\ Assistant at Digital Technologies department, \\ Voronezh State University \\ Russia, 394018, Voronezh, Universitetskaya sq., 1, VSU. \\ E-mail: evg-kisel2006@yandex.ru
}

Received 22.07.2019, revised 05.10.2020.

In this paper a generalization of the method of moments which was proposed in a recent article for modeling electrocardiogram waves with sets of several Gauss functions is performed. The purpose is to make the method applicable to functions of a more general form, while maintaining the simplicity of its program implementation. For this a series of mathematical transformations were carried out in a general form and sufficiently simple relations were obtained for calculating the parameters of the model signal. This makes it possible to use functions of various types for the approximation of signal regions, dictated by their physical model. The only restriction for the functions used is the existence of the required number of moments, and the moment of zero order must be different from zero. This paper demonstrates several examples of the implementation of the generalized method of moments. It is shown that in practice, depending on the type of function used for modeling, a number of computational features arise concerning the accuracy of the method and its stability with respect to noise. Obtained results can be useful for developing new effective models of biomedical signals, atomic and nuclear spectra, as well as other types of signals that have peak shaped local features.

Keywords: method of moments, digital signal, component separation, compression. 


\section{Citation}

Minin L.A., Suponev E.G., Kiselev E.A., "Using the method of moments for the approximation of signals containing peak shaped structural elements", Vestnik TvGU. Seriya: Prikladnaya Matematika [Herald of Tver State University. Series: Applied Mathematics], 2020, № 3, 68-77(in Russian). https://doi.org/10.26456/vtpmk576

\section{References}

[1] Addison P., "Wavelet Transforms and the ECG: a Review", Physiological Measurement, 26:5 (2005), R155-R199.

[2] Golubov B.I., Efimov A.V., Skvortsova V.A., Ryady i preobrazovaniya Uolsha: Teoriya i primeneniya, LKI Publ., Moscow, 2008 (in Russian), 352 pp.

[3] Bracewell R., The Fourier Transform and its Applications, McGraw-Hill, New York, 2000, 618 pp.

[4] Nikiforov P.L., "Model of an electrocardiographic signal based on a set of bell pulses", Vestnik molodykh uchenykh. Seriya: Tekhnicheskie nauki [Bulletin of Young Scientists. Series: Technical Sciences], 1998, №1, 64-68 (in Russian).

[5] Naser N., "Approximation of peaks in electrocardiograms by a combination of shifts of the Gaussian function", Sistemy upravleniya i informatsionnye tekhnologii [Management systems and information technologies], 2015, №1(59), 77-80 (in Russian).

[6] Kiselev E.A., Naser N.M., Suponev E.G., "The combined algorithm of compression of electrocardiogram signal using the Daubechies bursts and of the Gauss function", Sistemy upravleniya i informatsionnye tekhnologii [Management systems and information technologies], 2017, № 3(69), 53-56 (in Russian).

[7] Matulis V.E., Matulis V.E., Ivashkevich O.A., Prikladnaya kvantovaya khimiya [Applied quantum chemistry], BGU Publ., Minsk, 2007 (in Russian), 143 pp.

[8] Dobeshi I., Desyat lektsij po vejvletam [Ten Wavelet Lectures], Regular and Chaotic Dynamics Publ., Izhevsk, 2001 (in Russian), 464 pp.

[9] Zhuravlev M.V., Minin L.A., Sitnik S.M., "On the computational characteristics of interpolation by using the integer shifts of Gaussian functions", Vedomosti Belgorodskogo gosudarstvennogo universiteta [Bulletin of the Belgorod state University], 2009, № 13(68), 89-99 (in Russian).

[10] Lang K.R., Astrophysical Formulae, Springer Verlag, Berlin, Heidelberg, New York, 1980, $783 \mathrm{pp}$.

[11] Mukhin K.N., Eksperimentalnaya yadernaya fizika [Experimental nuclear physics]. V. 2, Lan Publ., SPb., 2009 (in Russian), 326 pp. 